\title{
Identification et comparaison des composés volatils des fruits de huit variétés de dattes marocaines
}

\author{
Hasnaâ HARRAK $^{a *}$, Max ReYNes ${ }^{b}$, Marc Lebrun ${ }^{b}$, Allal HAMOUdA ${ }^{c}$, Pierre Brat ${ }^{b}$
}

\begin{abstract}
a Unité de recherche Amélioration des plantes et de la qualité, Inra, BP 533, Marrakech 40000, Maroc hasnaa_harrak@hotmail.com

b Département des

Productions fruitières et horticoles, Cirad, BP 5035, 34032 Montpellier Cedex 1, France

${ }^{c}$ Département de Statistique et Informatique appliquées, IAV Hassan II, BP 6202, Rabat-Instituts, Maroc
\end{abstract}

* Correspondance et tirés à part

Reçu le 9 décembre 2004 Accepté le 21 juin 2005

Fruits, 2005, vol. 60, p. 267-278 (C) 2005 Cirad/EDP Sciences All rights reserved

DOI: $10.1051 /$ fruits:2005033

RESUMEN ESPAÑOL, p. 278

\section{Identification and comparison of volatile components of fruits of eight Moroccan date varieties.}

Abstract - Introduction. The identification of the volatile components of dates makes it possible to assess their organoleptic quality. This information is of technological interest to the agro-industrialists for processing dates and producing flavour extracts from low quality varieties, thereby increasing their commercial value. Our study aimed to identify the volatile components responsible for the flavour of eight varieties of Moroccan dates. A statistical classification of the varieties, based on their aromatic profiles, and a comparison with the results of previous work on the date flavour components were also carried out. Materials and methods. The volatile components were extracted by the dynamic headspace method and microwave desorption, and identified by gas chromatography-mass spectrometry. The data were analysed by simple correspondence analysis and hierarchic classification. Results and discussion. Forty-seven components were identified, of which 23 have not been found before in dates. Five components were found in all the varieties: 2,3-pentanedione, 2-methyl-butanal, hexanal, n-pentanol and limonene, while some varieties presented specific compounds. For instance, the Aziza variety was distinguished from the others by the presence of cyclobutanol, $p$-cymene and 1,3-dimethyl-benzene. Cyclopentadecadien-1-one, ethanol and geraniol were identified in the varieties Najda, Mejhoul and Boufeggous, respectively. The Bouskri and Iklane varieties were characterised by highly similar volatile profiles. Conclusion. Differences in the aromatic profiles of eight Moroccan date varieties were determined and the specific volatile components identified.

Morocco / Pboenix dactylifera / varieties / fruits / volatile compounds / aroma (organoleptic property)

\section{Identification et comparaison des composés volatils des fruits de huit} variétés de dattes marocaines.

Résumé - Introduction. L'identification des composés d'arôme des dattes permet d'apprécier leur qualité organoleptique ; elle revêt en outre un intérêt technologique en guidant les industriels dans certains processus de transformation du fruit et de production d'extraits d'arômes à partir des variétés de faible qualité, augmentant ainsi leur valeur marchande. Notre étude a eu pour objectif d'identifier des composés volatils responsables de l'arôme de huit variétés de dattes marocaines. Une classification statistique des variétés étudiées, basée sur leurs profils aromatiques, et une comparaison avec les résultats de travaux antérieurs effectués sur les composés d'arôme des dattes ont été également réalisées. Matériel et méthodes. Les composés volatils ont été analysés par la technique de piégeage dynamique de l'espace de tête et par désorption au micro-onde, puis ils ont été conjointement identifiés par chromatographie en phase gazeuse-spectrométrie de masse. L'étude statistique des données a été réalisée par analyse factorielle des correspondances et par classification hiérarchique ascendante. Résultats et discussion. Quarante-sept composés ont été identifiés dont vingt-trois non identifiés auparavant dans la datte. Cinq composés : la 2,3pentanedione, le 2-méthyl-butanal, l'hexanal, le n-pentanol et le limonène se sont révélés être communs à toutes les variétés, tandis que certains composés ont été caractéristiques d'une variété particulière. La variété 'Aziza' s'est distinguée par la présence de cyclobutanol, de $p$-cymène et de 1,3-diméthyl-benzène. Le cyclopentadécadièn-1-one, l'éthanol et le géraniol ont été identifiés respectivement dans les variétés 'Najda', 'Mejhoul' et 'Boufeggous'. En revanche, les variétés 'Bouskri' et 'Iklane' ont présenté une grande similarité de leurs profils aromatiques. Conclusion. Des différences de profils aromatiques entre les huit variétés étudiées ont été mises en évidence et des composés volatils "spécifiques" ont pu être identifiés.

Maroc / Pboenix dactylifera / variétés / fruits / composés volatils / profils aromatiques 


\section{Introduction}

La palmeraie marocaine, constituée de 4,7 millions d'arbres [1], représente un patrimoine très riche et diversifié du palmier dattier (Phonix dactylifera). Elle renferme environ $55,6 \%$ de "khalts" (clones non sélectionnés, issus de semis naturels), le reste étant composé de 223 variétés répertoriées [1, 2].

Les principales variétés marocaines présentent des caractéristiques physiques, physicochimiques et biochimiques très diversifiées leur conférant des qualités nutritionnelles, organoleptiques, technologiques et marchandes très diverses [1, 3, 4]. La variété 'Mejhoul' est considérée comme la meilleure datte au Maroc et jouit d'une renommée mondiale. Les variétés 'Boufeggous', 'Jihel' et 'Bouskri' ont une bonne valeur marchande. La variété 'Najda' est réputée pour les bonnes performances physiques de ses fruits, tandis que les variétés 'Aziza' et 'Bousthammi noire' sont très appréciées pour leur goût par les populations autochtones des palmeraies de l'Oriental et du Drâa. Ces variétés de dattes se caractérisent également par des aptitudes à la transformation très intéressantes : production de jus à partir des variétés 'Bousthammi noire' et 'Iklane' ou production de farines à partir des variétés 'Jihel' et 'Bouskri' [3, 4].

Cependant, aucune étude n'a encore porté sur les arômes des dattes marocaines. Or, l'identification de ces composés permet l'appréciation de la qualité organoleptique des fruits ; elle revêt en outre un intérêt technologique en guidant les industriels dans certains processus de transformation du fruit et de production d'extraits d'arômes à partir des variétés de faible qualité, augmentant ainsi leur valeur marchande [5].

Par ailleurs, les études tant qualitatives que quantitatives réalisées sur des dattes produites dans d'autres pays phoenicicoles sont également rares [6]. Dans une étude sur les composés volatils des dattes de la variété irakienne 'Zahdi', Jaddou et al. [7] ont pu séparer et identifier 11 acides gras libres volatils et 27 composés neutres appartenant aux classes des hydrocarbures saturés et insaturés, des aldéhydes, des alcools, des cétones et des phénols.

Barreveld [8] a rapporté que les acides organiques non volatils, comme les acides citrique, malique ou oxalique auraient une influence sur la flaveur des dattes fraîches. Ces acides sont présents en quantité non négligeable durant les phases de maturation des dattes et leur quantité diminue considérablement en fin de maturation. Reynes et al. [5] ont pu mettre en évidence 36 molécules contribuant à l'arôme de dattes de trois variétés tunisiennes ('Alligh', 'Deglet-Nour' et 'Kentichi'). Ils ont montré également que l'arôme fruité de ces dattes serait principalement dû à la présence d'alcools, d'aldéhydes et de cétones et ont conclu que ces composés pourraient être un outil de classification des diverses variétés.

Notre étude a eu pour objectif d'identifier des composés volatils responsables de l'arôme de huit variétés de dattes marocaines. Une classification statistique des variétés étudiées, basée sur leurs profils aromatiques, et une comparaison avec les résultats de travaux antérieurs effectués sur les composés d'arôme des dattes ont été également réalisées.

\section{Matériel et méthodes}

\subsection{Matériel végétal}

Huit variétés de dattes marocaines produites pendant la campagne 2000 ont été retenues pour cette étude : Aziza, Boufeggous, Bouskri, Bousthammi noire, Iklane, Jihel, Mejhoul et Najda. Ces dattes proviennent de deux régions du Maroc : la palmeraie du Drâa au sud du Grand-Atlas (Zagora) et la palmeraie de l'Oriental au sud-est du Moyen-Atlas (Figuig). Le choix de ces variétés a été motivé par le grand nombre de palmiers disponibles pour ces variétés, leur importance économique, leur bonne appréciation par le consommateur ou encore leur bonne aptitude technologique. Les dattes ont été récoltées en fin de maturation et n'ont subi aucun traitement.

\subsection{Méthodes analytiques}

Avant leur analyse, les dattes ont été parées (élimination des calices et des noyaux). Un échantillon de $100 \mathrm{~g}$ de pulpe de dattes a été finement broyée avec un Waring Blendor ${ }^{\circledR}$ (Waring, US) pendant $10 \mathrm{~min}$ avec $400 \mathrm{~mL}$ d'eau distillée refroidie à $4{ }^{\circ} \mathrm{C}$. Le système 
de piégeage de la technique d'analyse de l'espace de tête a consisté en l'introduction de $20 \mathrm{~mL}$ de purée de dattes dans un flacon en verre de $100 \mathrm{~mL}$ à col rodé. Le récipient a ensuite été fermé par une tête rodée de flacon Durand laveur, dont le tube interne a été plongé dans le broyat et le barbotage de l'hélium a été assuré à raison de $20 \mathrm{~mL} \cdot \mathrm{min}^{-1}$. Un piège Rektorik ${ }^{\circledR}$ en céramique, contenant un mélange à base de charbon actif permettant le piégeage des composés d'arôme, a été fixé sur le tube de sortie du flacon laveur. L'ensemble a été thermostaté à $36^{\circ} \mathrm{C}$ pendant $1 \mathrm{~h}$. Le contenu du piège a ensuite été désorbé à l'aide d'un injecteur à désorption micro-ondes (injecteur Rektorik ${ }^{\circledR}$ ) réglé à la puissance 5 (échelle de puissance arbitraire) et injecté dans une colonne capillaire de chromatographie en phase gazeuse DBWAX de $60 \mathrm{~m}$ de longueur, $0,32 \mathrm{~mm}$ de diamètre et 0,25 m d'épaisseur de film (J \& W Scientific, Folsom, CA) avec une division de 1/15 et un débit d'hélium de $1,5 \mathrm{~mL} \cdot \mathrm{min}^{-1}$. La température a été programmée à $50^{\circ} \mathrm{C}$ en isotherme pendant $6 \mathrm{~min}$, puis elle a été augmentée à raison de $6^{\circ} \mathrm{C} \cdot \mathrm{min}^{-1}$ jusqu'à atteindre $200^{\circ} \mathrm{C}$. L'injecteur et le détecteur ont été chauffés à $210^{\circ} \mathrm{C}$. L'acquisition des données et leur traitement ont été réalisés à l'aide du logiciel Chrom-Card (Thermo Separation Products). L'identification a été réalisée par le couplage chromatographie en phase gazeuse et spectrométrie de masse (CPG-SM). Ce système est un matériel Fisons, Modèle GC 8000 couplé à un spectromètre de masse Fisons Trio 1000 travaillant en impact électronique à $70 \mathrm{eV}$ avec une gamme de masses de (40 à 400) unités de masse atomique. L'identification des composés d'arôme par analyse des spectres de masse a été réalisée à l'aide de la banque de données Wiley (éd.), 1993, et du logiciel d'acquisition Labbase 2.1. Une comparaison des temps de rétention des pics obtenus avec des témoins purs a été également réalisée. Les composés d'arôme ont été analysés en effectuant deux répétitions.

\subsection{Analyses statistiques}

Une première classification ascendante hiérarchique des huit variétés de dattes marocaines a été réalisée en se basant sur le nombre de composés d'arôme de chaque classe chimique renfermée par chaque variété. Ensuite, le traitement statistique a consisté à tester la présence et l'absence de chaque composé d'arôme dans chaque variété en les codant respectivement par les valeurs 1 et 0 . Cette matrice de données a été d'abord dédoublée pour faciliter l'interprétation [9] : à chaque combinaison d'une variété et d'un composé d'arôme, on a fait correspondre deux valeurs valant respectivement 1 et 0 si le composé est présent dans la variété, et 0 et 1 si le composé est absent. Ces données ont été ensuite soumises à une analyse factorielle des correspondances (AFC) pour étudier les proximités entre les variétés et les composés d'arôme. Enfin, une classification ascendante hiérarchique a été réalisée sur les coordonnées des variétés obtenues sur les cinq premiers axes de l'AFC, afin de compléter les résultats portant sur l'identification de variétés présentant des profils aromatiques proches. Les calculs ont été effectués à l'aide du logiciel statistique Minitab [10].

\section{Résultats et discussion}

\subsection{Composition volatile des dattes}

À l'issue des analyses effectuées, une liste de 47 composés volatils identifiés dans les dattes des huit variétés marocaines étudiées a été constituée (tableau I) ; elle a été complétée par les temps de rétention respectifs de ces composés et par les propriétés sensorielles de certains d'entre eux, telles que décrites dans la littérature. Comparés aux travaux précédents de Jaddou et al. [7] pour la variété irakienne Zahdi, de Reynes et al. [5], Reynes [11] et Reynes et al. [12] pour les variétés tunisiennes Alligh, Deglet-Nour et Kentichi, et de Torres et al. [13] pour des dattes cultivées en Espagne (tableau II), il ressort que vingt-trois composés semblent spécifiques des variétés marocaines étudiées.

Quatre classes chimiques forment l'essentiel des 47 composés volatils identifiés dans cette étude : les alcools, les aldéhydes, les cétones et les hydrocarbures. Par rapport aux études antérieures, on peut noter que Reynes et al. [5], en utilisant les méthodes de l'extraction au pentane et de piégeage par espace de tête, n'ont pas identifié d'hydrocarbures parmi les classes majoritaires des 


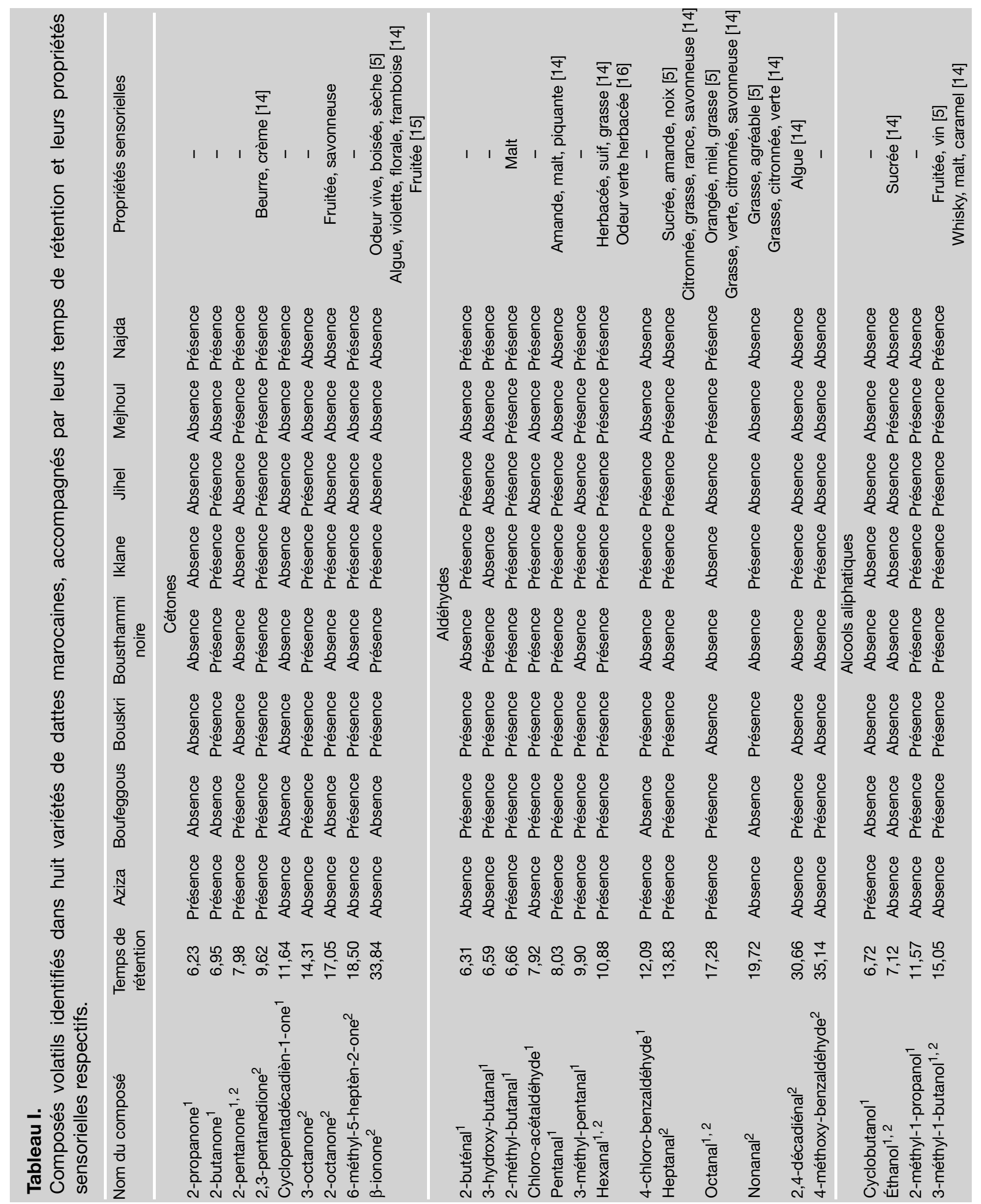




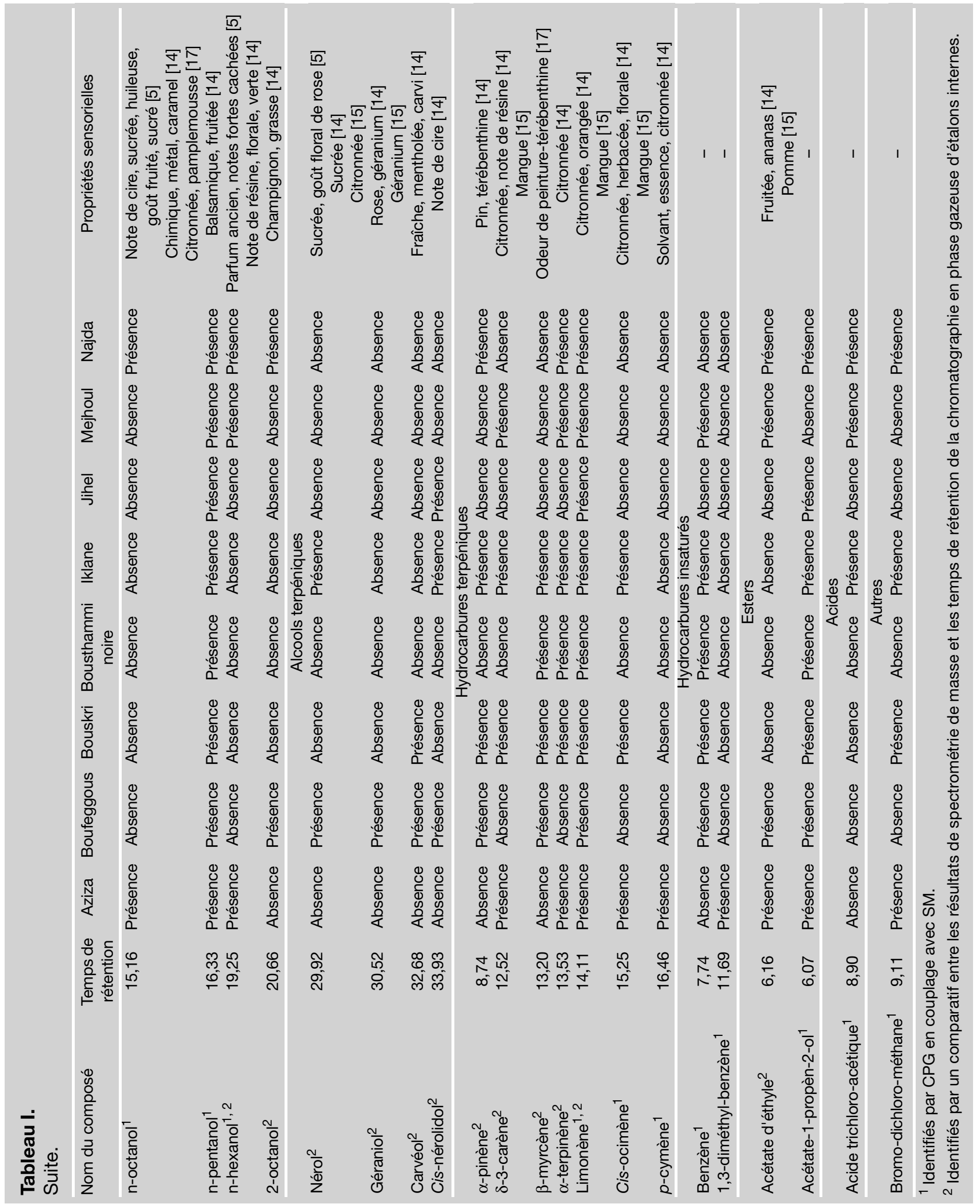


Tableau II.

Composés volatils des dattes, tels que décrits par la littérature, regroupés en classes chimiques.

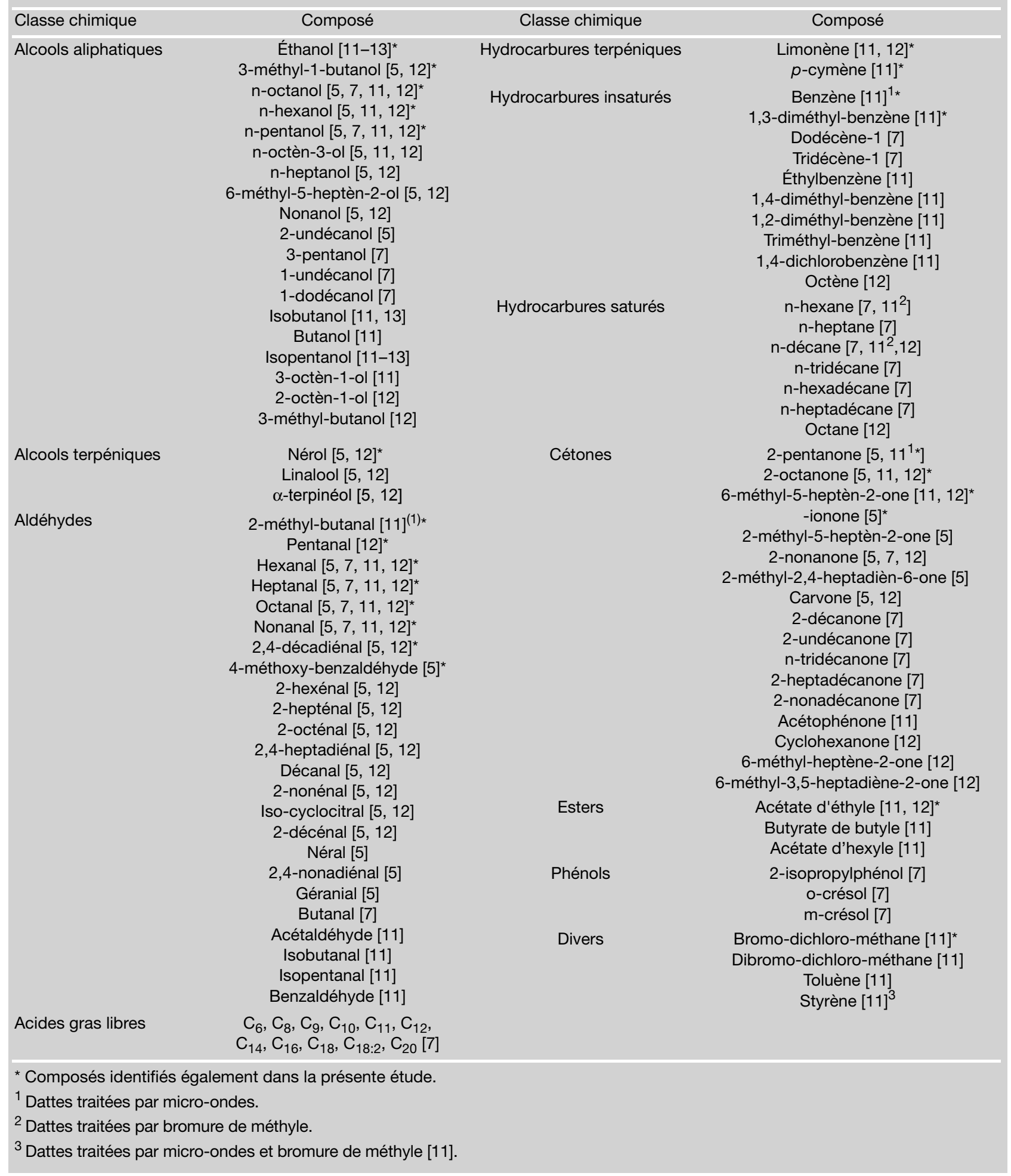




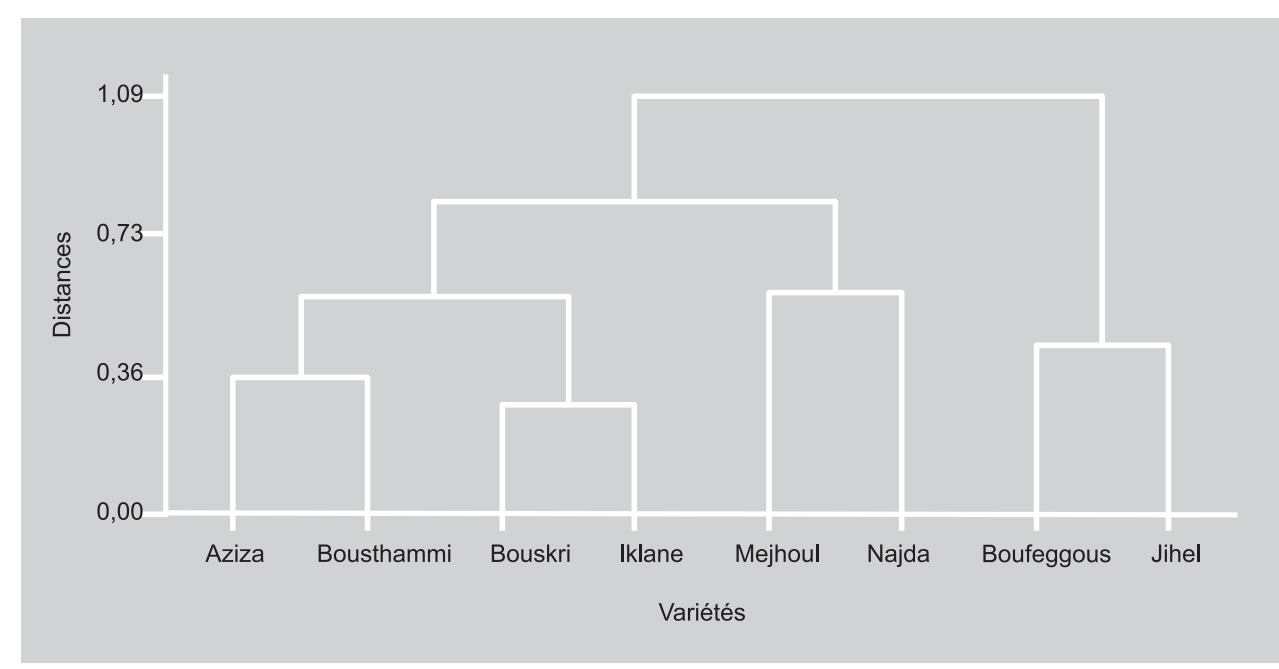

composés volatils, et que Torres et al. [13] ont identifié 25 composés appartenant principalement aux alcools et aux aldéhydes, tandis que Jaddou et al. [7], en utilisant les méthodes de distillation à basse température et à pression réduite et la chromatographie en phase gazeuse, ont identifié des acides gras libres volatils et des phénols.

Il apparaît également d'importantes différences entre les composés d'arôme spécifiques identifiés dans les dattes marocaines et ceux rencontrés dans la littérature. Les principales différences concernent le nombre de composés terpéniques (alcools et hydrocarbures) supérieur dans le profil aromatique des huit variétés marocaines : trois alcools terpéniques (géraniol, carvéol, cisnérolidol) et cinq hydrocarbures terpéniques ( $\alpha$-pinène, $\delta$ - 3 -carène, $\beta$-myrcène, $\alpha$-terpinène, cis-ocimène) sont nouvellement identifiés (tableau I). De même, trois nouveaux alcools aliphatiques (cyclobutanol, 2-méthyl-1propanol, 2-octanol), cinq nouveaux aldéhydes (2-buténal, 3-hydroxy-butanal, chloroacétaldéhyde, 3-méthyl-pentanal, 4-chlorobenzaldéhyde) et cinq nouvelles cétones (2-propanone, 2-butanone, 2,3-pentanedione, cyclopentadécadièn-1-one, 3-octanone) ont été identifiés.

\subsection{Comparaison des variétés sur la base des classes chimiques des composés volatils}

À partir des composés volatils identifiés dans les huit variétés de dattes marocaines étudiées (tableau I) et du dendrogramme établi sur la base du nombre de composés volatils de chaque classe chimique (figure 1), il peut être constaté qu'il existe une grande ressemblance : sont relativement les plus riches en hydrocarbures terpéniques (six composés), très riches en aldéhydes (dix et onze composés, respectivement) et en composés cétoniques (six composés), et les moins riches en alcools aliphatiques (trois composés),

- entre les variétés Aziza et Bousthammi noire qui contiennent, notamment, moins de composés cétoniques (quatre composés) et qui sont dépourvues d'alcool terpénique.

La variété Boufeggous se caractérise par le plus grand nombre d'alcools terpéniques (quatre composés) et par un grand nombre d'aldéhydes (onze composés), ce qui l'oppose notamment aux variétés Aziza, Mejhoul, Najda et Bousthammi noire qui sont dépourvues d'alcools terpéniques et qui présentent un nombre relativement faible d'aldéhydes. La variété Mejhoul se différencie par une faible présence de composés cétoniques (deux composés) et d'hydrocarbures terpéniques (trois composés) et par une plus forte présence d'alcools aliphatiques (cinq composés), et, de ce point de vue, elle s'oppose aux variétés Bouskri et Iklane ; la variété Mejhoul se caractérise également par l'absence d'alcools terpéniques, tout comme la variété Najda qui est, elle, cependant, riche en cétones et en alcools aliphatiques.
- entre les variétés Bouskri et Iklane qui
Figure 1.

Dendrogramme permettant de classer huit variétés de dattiers marocains, obtenu par une classification ascendante hiérarchique basée sur le nombre de composés volatils recensés dans différentes classes chimiques. 
Les différentes classes chimiques de composés volatils n'ont pas la même contribution à l'arôme de la datte. Jaddou et al. [7] ont indiqué que les hydrocarbures aliphatiques seraient probablement d'une importance secondaire dans l'arôme de ce fruit. Dans notre étude, sept hydrocarbures terpéniques ont été identifiés : $\alpha$-pinène, $\delta$ - 3 carène, $\beta$-myrcène, $\alpha$-terpinène, limonène, cis-ocimène et $p$-cymène. Ces composés sont caractérisés notamment par des notes citronnées (tableau I). Les composés carbonylés comme les alcools, les aldéhydes et les cétones sont des composés extrêmement importants, qui participent à de très nombreuses notes (fruitée, florale, citronnée) [15]. Les aldéhydes apportent généralement des notes fruitées [17], mais les aldéhydes aliphatiques à six ou neuf atomes de carbones (hexanal, nonénal) sont responsables d'odeurs vertes et herbacées [16]. Les alcools terpéniques ou non apportent une grande diversité de notes olfactives : notes herbacée, fruitée, citronnée, florale et fongique [15].

Les esters apportent essentiellement des notes fruitées, plus ou moins beurrées, et qui sont très souvent caractéristiques d'un arôme particulier [15]. Pour les huit variétés marocaines, deux esters ont été identifiés : l'acétate d'éthyle et l'acétate-1-propèn-2-ol.

Crouzet [16] a rapporté trois classes de mécanismes impliqués dans la formation des composés volatils dans les fruits : biosynthèse, réactions enzymatiques directes et réactions enzymatiques indirectes, en considérant qu'il existe des arômes primaires qui sont ceux du fruit intact (classe I) et des arômes secondaires qui se forment lors de la destruction des cellules (correspondant approximativement aux classes II et III). Parmi ces mécanismes, on a souligné l'importance, pour l'arôme des fruits, des métabolismes des acides gras, des terpènes, des acides aminés et des glucides dans les principales voies conduisant à la biosynthèse des composés d'arôme [16]. Les aldéhydes aliphatiques à six ou neuf atomes de carbones (hexanal, nonénal) sont formés par action de la lipoxygénase sur les acides gras polyinsaturés linoléique et linolénique. On a montré également que le $\beta$-carotène se transforme au cours de la fermentation du thé en $\beta$-ionone et différents composés non iden- tifiés. Cette réaction ne se produit pas si les enzymes du thé sont inactivés thermiquement, ce qui permet de penser que se sont les flavonols oxydés qui seraient responsables de la dégradation des caroténoïdes [16]. La $\beta$-ionone, caractérisant les fruits rouges et possédant une note fruitée [16], a été identifiée dans trois des variétés étudiées, dont deux d'entre elles sont de couleur brun-rougeâtre (Bousthammi noire et Iklane). Ces deux variétés se caractérisent durant le troisième stade de maturation (stade Khalal) par une couleur rouge [3, 4]. Les hydrocarbures saturés pourraient être produits à partir de la fraction lipidique de la datte [7]. Le mécanisme de formation de la plupart des alcools de la datte pourrait impliquer la décomposition des hydropéroxydes des acides gras insaturés, alors que d'autres alcools pourraient aussi être formés par réduction des composés carbonylés présents dans l'arôme de la datte [7]. Les aldéhydes légers pourraient être formés par l'auto-oxydation des acides gras insaturés [7].

\subsection{Distribution des composés d'arôme spécifiques}

Indépendamment de leurs classes chimiques, les composés d'arôme ont pu être regroupés en fonction du nombre de variétés dans lesquelles chacun d'eux était présent (tableau III). Seuls cinq composés sont apparus être communs aux huit variétés : la 2,3-pentanedione, le 2-méthyl-butanal, l'hexanal, le n-pentanol et le limonène. À l'opposé, quinze composés sont plutôt rares, chacun ne se trouvant tout au plus que dans deux variétés. Enfin, des composés spécifiques ont pu être identifiés : le cyclopentadécadièn-1-one pour la variété Najda, l'éthanol pour la variété Mejhoul, le géraniol pour la variété Boufeggous, et le $p$-cymène, le 1,3diméthyl-benzène et le cyclobutanol pour la variété Aziza. Vingt-sept composés, soit $57 \%$ du total, sont apparus communs à au moins quatre variétés (tableau III).

Il ressort de la matrice des occurrences et des cooccurrences (tableau IV) indiquant le nombre de composés présents dans la datte de chaque variété (éléments diagonaux) et le nombre de composés présents simultanément dans les dattes de chaque couple de 
Tableau III.

Répartition des composés d'arôme dans les fruits de huit variétés marocaines de palmiers dattiers.

Cyclopentadécadièn-1-one, cyclobutanol, éthanol, géraniol, $p$-cymène, 1,3-diméthyl-benzène

2-propanone, 2-octanone, nonanal, 2,4-décadiénal, 4-méthoxy-benzaldéhyde, n-octanol, 2-octanol, nérol, carvéol

$\beta$-ionone, $n$-hexanol, cis-nérolidol, cis-ocimène, acide trichloro-acétique

2-pentanone, 6-méthyl-5-heptèn-2-one, 3-hydroxy-butanal, 4-chloro-benzaldéhyde, octanal, $\alpha$-pinène,

$\delta$-3-carène, $\beta$-myrcène, acétate d'éthyle, bromo-dichloro-méthane

3-octanone, 2-buténal, chloro-acétaldéhyde, benzène

2-butanone, pentanal, 3-méthyl-pentanal, heptanal, 2-méthyl-1-propanol, $\alpha$-terpinène

3-méthyl-1-butanol, acétate-1-propèn-2-ol

2,3-pentanedione, 2-méthyl-butanal, hexanal, n-pentanol, limonène

\section{Tableau IV.}

Nombre de composés volatils par variété et nombre de composés présents simultanément dans les dattes de chaque couple de variétés (palmeraie marocaine).

$\begin{array}{lcccccccc}\text { Variété } & \text { Aziza } & \text { Boufeggous } & \text { Bouskri } & \text { Bousthammi } & \text { Ikane } & \text { Jihel } & \text { Mejhoul } & \text { Najda } \\ \text { Aziza } & 24 & 12 & 15 & 9 & 15 & 10 & 13 & 16 \\ \text { Boufeggous } & - & 29 & 20 & 14 & 22 & 13 & 13 & 17 \\ \text { Bouskri } & - & - & 29 & 17 & 27 & 14 & 12 & 16 \\ \text { Bousthammi } & - & - & - & 17 & 16 & 11 & 9 & 11 \\ \text { Ikane } & - & - & - & - & 32 & 15 & 13 & 16 \\ \text { Jihel } & - & - & - & - & - & 15 & 8 & 9 \\ \text { Mejhoul } & - & - & - & - & - & - & 18 & 13 \\ \text { Najda } & - & - & - & - & - & - & - & 25\end{array}$

variétés (éléments extérieurs à la diagonale) que les variétés Bousthammi noire, Jihel et Mejhoul sont les moins riches en composés volatils (entre 15 et 18 composés), alors que les variétés Boufeggous, Bouskri et Iklane sont les plus riches (entre 29 et 32 composés). Ces valeurs restent inférieures aux trente-cinq composés identifiés par Reynes [11] dans la datte de la variété tunisienne Alligh. Il apparaît également que $50 \%$ des couples de variétés n'ont en commun que neuf à treize composés d'arôme. Les variétés Bouskri et Iklane ont le plus grand nombre de composés en communs (27 composés).

L'AFC, réalisée sur la matrice de présence et d'absence des composés dans les variétés, permet de renseigner sur les différences de composition qualitative en composés volatils des huit variétés. La contribution à l'iner- tie totale expliquée par les cinq premiers axes a été de $89,8 \%$, dont 31,0\% pour l'axe 1 , $18,6 \%$ pour l'axe 2, 16,1\% pour l'axe 3, $12,4 \%$ pour l'axe 4 et 11,7\% pour l'axe 5 . L'analyse des projections des points-variétés dans le plan principal (figure 2) montre que l'axe 1 oppose les variétés Iklane et Bouskri aux variétés Aziza et Najda : les deux premières variétés sont caractérisées par la présence de $\beta$-myrcène, de 3 -octanone, de 2 -octanone, de nonanal, de $\beta$-ionone et de 2 -méthyl-1propanol et l'absence de 2-propanone, de n-hexanol et de n-octanol ; l'axe 2 sépare la variété Aziza des variétés Boufeggous et Najda qui sont elles-mêmes caractérisées par la présence de 3-méthyl-1-butanol et de 2-octanol et l'absence de 4-chloro-benzaldéhyde, de cyclobutanol, de cis-ocimène, de $p$-cymène et de 1,3-diméthyl-benzène. 


\section{H. Harrak et al.}

Figure 2.

Projections des points " variétés » dans le plan principal d'une analyse factorielle des la présence-absence de composés volatils identifiés dans les fruits de huit variétés de dattes marocaines. correspondances, basée sur

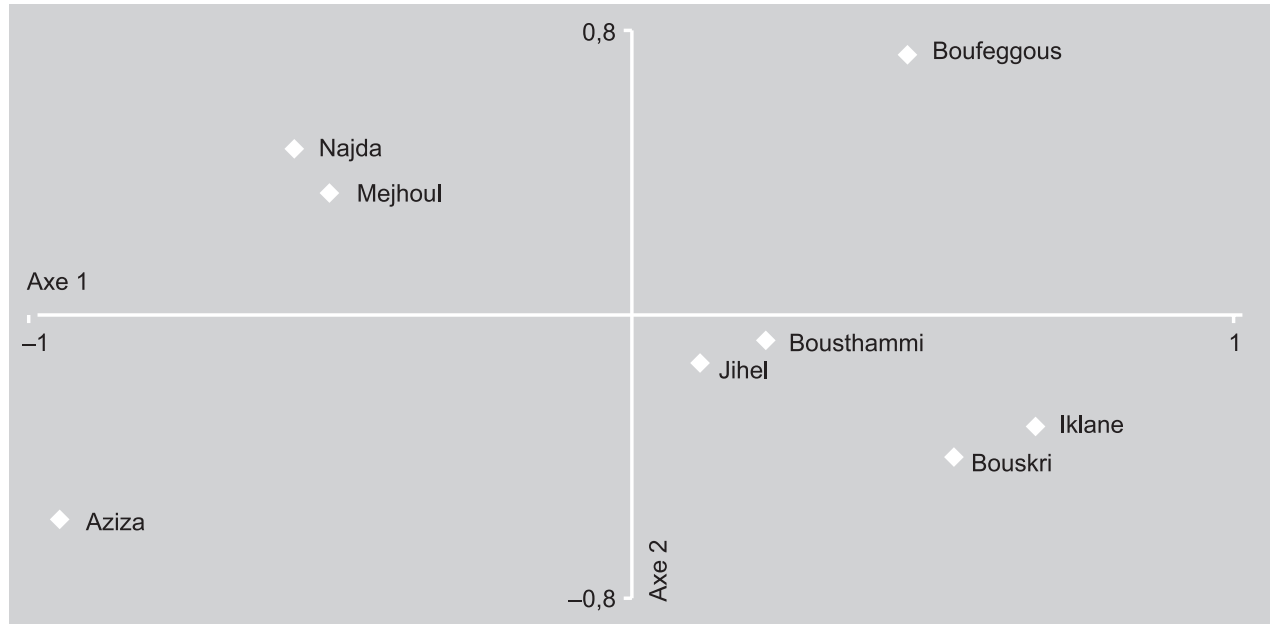

Figure 3.

Dendrogramme permettant de classer huit variétés de dattiers marocains, obtenu par une classification ascendante hiérarchique basée sur la présence-absence de composés volatils identifiés dans leurs fruits.

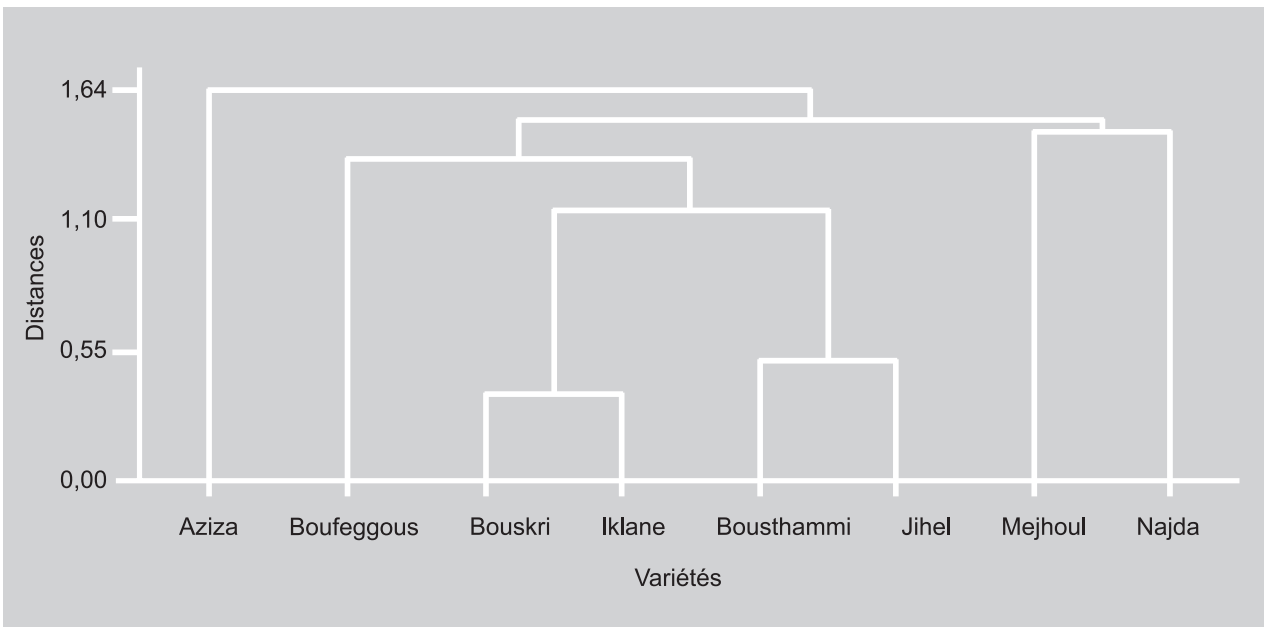

Les axes 3, 4 et 5 de la même AFC, non représentés sur la figure 2, ont aussi permis de séparer les variétés: l'axe 3 oppose la variété Najda aux variétés Jihel, Mejhoul et Bousthammi noire, caractérisées par l'absence des composés 2-propanone, cyclopentadécadièn-1-one, 6-méthyl-5-heptèn-2-one, n-octanol, 2-octanol, $\alpha$-pinène et bromo-dichlorométhane et la présence du composé 2-méthyl1-propanol ; l'axe 4 oppose la variété Boufeggous aux variétés Najda et Bousthammi noire, caractérisées par la présence du composé 2-butanone et l'absence des composés 2,4-décadiénal, 4-méthoxy-benzaldéhyde, heptanal, nérol et géraniol. L'axe 5 sépare la variété Mejhoul et les variétés Jihel et Boufeggous, caractérisées par la présence des composés pentanal, cis-nérolidol, acétate-1propèn-2-ol et l'absence des composés éthanol, $\delta$ - 3 -carène, $\alpha$-terpinène et acide trichloro-acétique.

Ces résultats se confirment par la classification ascendante hiérarchique qui met en évidence les fortes différences existant entre les profils aromatiques des huit variétés (figure 3). À noter, cependant, le relatif rapprochement, d'une part entre les variétés Bouskri et Iklane avec vingt-sept composés en commun, d'autre part entre les variétés Jihel et Bousthammi noire qui présentent les plus faibles nombres de composés volatils (respectivement 15 et 17 composés). La variété Aziza, par ailleurs, se distingue par 
la présence de trois composés spécifiques (le cyclobutanol, le p-cymène et le 1,3-diméthyl-benzène) et par l'absence de 3-méthyl1-butanol.

\section{Conclusion}

Parmi les quarante-sept composés volatils identifiés dans les huit variétés de dattes marocaines, vingt-trois n'avaient pas été identifiés précédemment dans les dattes des autres pays phoenicicoles comme l'Irak, la Tunisie et l'Espagne. Cinq composés ont été identifiés dans toutes les variétés étudiées : le 2-méthyl-butanal, la 2,3-pentanedione, l'hexanal, le limonène et le n-pentanol. Le nombre de composés volatils par variété a été assez variable : de quinze composés pour la variété Jihel à trente-deux composés pour la variété Iklane.

Nous avons aussi pu mettre en évidence de fortes différences de composition en composés volatils entre les variétés. Le classement des variétés a varié selon que leur comparaison a été faite sur la base de la présence-absence de chaque composé ou sur la base de la représentation des différentes classes chimiques des composés dans les variétés. Toutefois, du point de vue de la nature chimique des composés, certaines similarités ont été notées : les variétés Bouskri et Iklane ont présenté la caractéristique d'être les plus riches en hydrocarbures terpéniques, très riches en aldéhydes et en composés cétoniques et les moins riches en alcools aliphatiques, alors que les variétés Aziza et Bousthammi noire se sont révélées contenir notamment le même nombre de composés cétoniques et être dépourvues d'alcools terpéniques. Les variétés Boufeggous et Mejhoul, très appréciées par le consommateur marocain et de grande valeur marchande, se sont différenciées l'une par une relative diversité des composés d'alcools terpéniques, l'autre par le plus faible nombre de composés cétoniques.

Par ailleurs, certains composés volatils sont connus pour avoir de faibles seuils de perception et ils pourraient être très impliqués dans l'arôme caractéristique des dattes (la 2,3-pentanedione, par exemple, qui a un seuil de $5 \mu \mathrm{g} \cdot \mathrm{kg}^{-1}$ dans l'eau [18]). De même, d'autres composés présents à des concen- trations largement supérieures à leurs seuils de perception peuvent également être importants pour l'arôme des dattes. Une étude ultérieure sera nécessaire pour déterminer les composés d'impact de l'arôme des dattes ainsi que les mécanismes impliqués dans leur formation. Il sera aussi intéressant d'étudier les effets de paramètres autres que la variété, tels que le degré de maturité et les possibles "effets terroirs", qui pourraient mettre en évidence une éventuelle influence des zones de production sur la diversité des composés volatils des dattes.

\section{Remerciements}

Nous remercions le réseau de Recherches et développement du palmier dattier (sousréseau Technologies post-récolte des dattes) (ACSAD/FADES/BID) et le projet RAB 98G31 (IPGRI/PNUD/FEM) “ Gestion participative des ressources génétiques du palmier dattier dans les oasis du Maghreb " pour leur contribution à la réalisation de cette étude. Nos remerciements vont également à l'équipe du domaine expérimental Nebch de l'Inra à Zagora (Maroc).

\section{Références}

[1] Sedra M.H., El Filali H., Nour S., Boussak Z., Benzine A., Allaoui M., La palmeraie dattière marocaine : évaluation du patrimoine phénicicole, Fruits 51 (1996) 247-259.

[2] Toutain G., Bachra A., Chari A., Cartographie variétale de la palmeraie marocaine, Direction de la recherche agronomique, Rabat, Maroc, 1971.

[3] Harrak H., Étude des aptitudes technologiques des principales variétés de dattes marocaines : caractérisation physique, physicochimique et biochimique, Inra, Marrakech, Maroc, Mém., 1999, 156 p.

[4] Harrak H., Boujnah M., Hamouda A., Caractérisations physiques et morphologiques des principales variétés de dattes marocaines, $\mathrm{Al}$ Awamia 107 (2003) 69-87.

[5] Reynes M., Lebrun M., Shaw P.E., Identification of volatile date components and use for multivariate analysis to distinguish date varieties, J. Food Quality 19 (1996) 505-514.

[6] Munier P., Le palmier-dattier, Coll. Techniques agricoles et productions tropicales, Maisonneuve et Larose, Paris, France, 1973. 
[7] Jaddou H., Mhaisen M.T., Al-Hakim M., Flavour volatile analysis of Zahdi dates by gasliquid chromatography, Date Palm J. 3 (2) (1984) 367-379.

[8] Barreveld W.H., Date palm products, FAO Agric. Serv. Bull., no. 101, Rome, Italy, 1993.

[9] Benzécri J. P., L'analyse des données, 2 vol., Dunod, Paris, France, 1973.

[10] Anon., Minitab reference manual, release 13, Minitab Inc., State College, Pennsylvania, USA, 2000.

[11] Reynes M., Influence d'une technique de désinfestation par micro-ondes sur les critères de qualité physico-chimiques et biochimiques de la datte, Institut National Polytechnique de Lorraine (INPL), Thèse, Nancy, France, 1997.

[12] Reynes M., Lebrun M., Shaw P., Identification des arômes de trois variétés de dattes, in : Ferry M., Greiner D., Bedrani S., Tonneau J.P. (Éds.), Le palmier dattier dans l'agriculture d'oasis des pays méditerranéens, CIHEAM, Paris, France, $\mathrm{n}^{\circ}$ 28, 1996, p. 212.

[13] Torres P., Reynes M., Lebrun M., Ferry M., Volatile constituent of dates from Phoenix dactylifera grown in Elche, in : Ferry M., Greiner D., Bedrani S., Tonneau J.P. (Éds.), Le pal- mier dattier dans l'agriculture d'oasis des pays méditerranéens, CIHEAM, Paris, France, $\mathrm{n}^{\circ} 28,1996$, p. 214.

[14] Acree T., Arn H., Flavonet, http://www.flavonet.org, ${ }^{\odot}$ Datu Inc., 2004.

[15] Richard H., Connaissance de la nature des arômes, in : Richard H., Multon J.L. (Éds), Les arômes alimentaires, Partie I. Généralités, Coll. Sciences et techniques agro-alimentaires, Lavoisier TEC \& DOC-Apria, Paris, France, 1992.

[16] Crouzet J., La biogenèse des arômes, in : Richard H., Multon J.L. (Éds), Les arômes alimentaires, Partie II. Différentes voies de synthèse, Coll. Sciences et techniques agro-alimentaires, Lavoisier TEC \& DOC-Apria, Paris, France, 1992.

[17] Guichard E., L'analyse sensorielle par " sniffing ", in : Richard H., Multon J.L. (Éds.), Les arômes alimentaires, Partie V. Méthodes d'analyse, Coll. Sciences et techniques agroalimentaires, Lavoisier TEC \& DOC-Apria, Paris, France, 1992.

[18] Milo C., Grosch W., Changes in the odorants of boiled trout (Salmo fario) as affected by the storage of the raw material, J. Agric. Food Chem. 41 (1993) 2076-2081.

\section{Definición y comparación de los compuestos volátiles de los frutos de ocho variedades de dátiles marroquíes.}

Resumen - Introducción. La definición de compuestos de aroma de dátiles permite apreciar su calidad organoléptica. Por otro lado, supone un interés tecnológico, ya que guía a los industriales en algunos procesos de transformación del fruto y de producción de extractos de aromas, a partir de variedades de baja calidad, aumentando así su valor mercantil. Nuestro estudio tuvo como objetivo identificar compuestos volátiles responsables del aroma de ocho variedades de dátiles marroquíes. Se realizaron también tanto una clasificación estadística de las variedades estudiadas, basada en los perfiles aromáticos de las mismas, como una comparación con los resultados de trabajos anteriores, que habían sido efectuados sobre los compuestos de aroma de dátiles. Material y métodos. Los compuestos volátiles fueron analizados mediante la técnica de instalación de trampas dinámica del espacio frontal y mediante desorción en el microondas, luego fueron definidos conjuntamente por cromatografía en fase gaseosa - espectrometría de masa. El estudio estadístico de los datos fue realizado mediante un análisis factorial de las correspondencias; y, mediante una clasificación jerárquica ascendente. Resultados y discusión. Se identificaron cuarenta y siete compuestos de los cuales veintitrés no habían sido definidos antes en el dátil. Cinco compuestos: el 2,3-pentanedione, el 2-metil-butanal, el hexanal, el npentanol y el limoneno resultaron ser comunes en todas las variedades, mientras que algunos compuestos resultaron ser característicos de una variedad particular. La variedad 'Aziza' se distinguió por la presencia de cyclobutanol, p-cimeno y 1,3-dimetil-beceno. El cyclopentadecadien1-one, el etanol y el geraniol se identificaron respectivamente en las variedades 'Najda', 'Mejhoul' y 'Boufeggous'. En cambio, las variedades 'Bouskri' y 'Iklane' presentaron una gran semejanza entre sus perfiles aromáticos. Conclusión. Se pusieron de manifiesto algunas diferencias de perfiles aromáticos entre las ocho variedades estudiadas y se pudieron identificar los compuestos volátiles "específicos".

Marruecos / Phoenix dactylifera / variedades / frutas / compuesto volátil / aroma (propiedad organoléptica) 